Original Article

\title{
OPTIMIZATION AND CHARACTERIZATION OF DOXORUBICIN LOADED SOLID LIPID NANOSUSPENSION FOR NOSE TO BRAIN DELIVERY USING DESIGN EXPERT SOFTWARE
}

\author{
VIRAG A. SHAH ${ }^{*}$, JAYVADAN K. PATEL ${ }^{2}$
}

${ }^{1}$ Hemchandracharya North Gujarat University, Patan, ${ }^{2}$ Nootan Pharmacy College, SPCE Campus, Visnagar, Gujarat 384315

Email: viragshah687@gmail.com

Received: 17 Feb 2021, Revised and Accepted: 25 Mar 2021

\begin{abstract}
Objective: The goal of the current study was to investigate the possible use of solid lipid nanosuspension (SLNs) as a drug delivery method to boost doxorubicin (DOX) brain-targeting performance after intranasal (i. n.) administration.

Methods: $3^{3}$ factorial design was applied for optimization by using lipid concentration, surfactant concentration, and High-speed homogenizer (HSH) stirring time as dependent variables, and their effect was observed on particles size, Polydispersity index (PDI), and entrapment efficiency.

Results: With the composition of Compritol® 888 ATO ( $4.6 \% \mathrm{w} / \mathrm{v})$, tween 80 (1.9\% w/v), and HSH stirring time, the optimized formula DOX-SLNs prepared $(10 \mathrm{~min})$. Particle size, PDI, zeta potential, entrapment efficiency, percent in vitro release were found to be $167.47 \pm 6.09 \mathrm{~nm}, 0.23 \pm 0.02$, $24.1 \mathrm{mV}, 75.3 \pm 2.79$, and $89.35 \pm 3.27$ percent in $24 \mathrm{~h}$, respectively, for optimized formulation (V-0). No major changes in particle size, zeta potential, and entrapping efficiency were found in the stability studies at $4 \pm 2{ }^{\circ} \mathrm{C}$ (refrigerator) and $25 \pm 2{ }^{\circ} \mathrm{C} / 60 \pm 5 \%$ RH up to $3 \mathrm{mo}$.
\end{abstract}

Conclusion: Following the non-invasive nose-to-brain drug delivery, which is a promising therapeutic strategy, the positive findings confirmed the current optimized DOX-loaded SLNs formulation.

Keywords: Solid lipid Nanosuspension, Homogenization and Ultrasonication, Characterization, Factorial design, Nose to brain delivery

(C) 2021 The Authors. Published by Innovare Academic Sciences Pvt Ltd. This is an open access article under the CC BY license (https://creativecommons.org/licenses/by/4.0/) DOI: https://dx.doi.org/10.22159/ijpps.2021v13i5.41137. Journal homepage: https://innovareacademics.in/journals/index.php/ijpps.

\section{INTRODUCTION}

Currently, the most widely used cancer treatment is through chemotherapy administered either by the intravenous or oral route, which harms the normal cells more than just the cancerous cells in the body and causes many unwanted side effects [1].

Doxorubicin hydrochloride (DOX) is a broad spectrum anthracycline anticancer drug administrated intravenously, including the US Food and Drug Administration (USFDA) approved pegylated liposomal doxorubicin marketed as Doxil $®$ for treatment of numerous human cancers [2-4]. No significant improvement in efficiency has been found through studies despite the use of Doxil $₫$ due to sluggish and passive release of the drug [5].

Treating brain diseases using hydrophilic drugs like DOX is yet more difficult as Blood-Brain Barrier (BBB) restricts the entry of drug and prevent their uptake in the brain. Due to BBB, no large molecule can reach the brain and less than $2 \%$ of small molecules find an entry in the brain, creating a huddle in the treatment of many lifethreatening brain diseases, including brain cancer [5].

Intranasal administration is a non-invasive way that can deliver drugs directly into the brain, bypassing BBB through the trigeminal or olfactory pathway. It has been exploited by researchers, followed by the advantages of self-medication, ease delivery, avert first-pass metabolism, rapid onset, reduction in dose amount when compared to oral route [6].

Nanoparticulate-based drug delivery may improve the drug delivery to the brain via nasal route. These systems protect the drug from Pglycoprotein (P-gp) efflux, enzyme, and/or chemical degradation with an increase in bioavailability and specific biodistribution [7].

Nanotechnology carriers include polymeric-based nanoparticles, magnetic-based nanoparticles, ligand-based nanoparticles, lipidbased nanoparticles, etc.

Solid Lipid Nanoparticles (SLN) is a submicron lipidic carrier entrapping the drug in the lipid core and stabilized with the aid of surfactant, co-surfactant, and/or stabilizer.

The objectives of the current study were to apply an experimental design approach in the development of DOX-loaded SLN delivery administered via nasal route. The experimental design was applied to optimize the independent variables (Concentration of Compritol 888 ATO, Concentration of tween 80, and High-Speed Homogenizer time) to achieve low particle size, low polydispersity index (PDI), and high entrapment efficiency (EE). DOX SLN were formulated, optimize, and were characterized for physicochemical, morphological, in vitro release, and kinetic mechanisms $[8,9]$.

\section{MATERIALS AND METHODS}

\section{Materials}

DOX was collected from Amneal Pharmaceuticals Ltd (Ahmedabad, India) as a gift sample. Apifil, Compritol 888 ATO, Compritol HD5 ATO, Capryol 90, and Precirol ATO 5 (PA) were collected as gift samples from Gattefosse Pvt. Ltd. (Mumbai, India). Stearic acid Tween 80, and Poloxamer-407 (Pol-407) were procured from Sigma-Aldrich (Bangalore, India). Hydrogenated soybean phosphatidylcholine (HSPC) 50 was collected from the Sun Pharma Advanced Research Centre (SPARC, Vadodara) as a gift sample.

Selection of lipid using Solubility study of DOX in different lipids

Lipid selection is one of the most significant factors in Solid Lipid Nanosuspension in deciding the drug's encapsulation performance in the lipid. The DOX solubility analysis has been investigated in five different lipids viz. Compritol 888 ATO, Compritol HD 5 ATO, Precirol ATO 5, Apifil, Stearic acid for determining their potential to solubilize DOX. The solubility could not be assessed by the equilibrium technique since most of the lipids used were in solid condition. Hence, an alternative approach was used, where DOX $(20 \mathrm{mg})$ was placed in five separate vials. The lipids were separately heated above their melting point and gradually added to the vials until a clear solution of lipids is formed. The experiment was performed in triplicate [10]

\section{Formulation of DOX loaded SLN}

SLN was formulated using the process of homogenization and ultrasonication. The procedure was consist of taking lipid and drug in one part while an aqueous solution of surfactant and stabilizer in another part. Drug and lipid mixture was melted above the melting point of lipid. An aqueous portion was heated at the same 
temperature. When both the parts attain an equilibrium, an aqueous portion was added incorporated into the lipid phase, followed by emulsification by a high-speed homogenizer. The temperature of the mixture was maintained constant until complete emulsification takes place. After obtaining primary emulsion, the mixture was ultrasonicated using an Ultrasonic homogenizer (Probe sonicator) (orchid scientific and innovative India Pvt ltd, Ambad, Nashik) to obtain Solid lipid-based Nanosuspension (SLNS).

\section{Optimization of variables by applying factorial design}

$3^{3}$ experimental design was used for the optimization and evaluation of the relationship between the independent variables [critical process parameters (CPP)] viz., X1= lipid concentration, X2= surfactant concentration, and X3= High-Speed Homogenizer (HSH) time and dependent variables (responses) [Critical Quality Attribute (CQA)] such as Y1= Particle size, Y2= Polydispersibility index (PDI) and $\mathrm{Y} 3=\%$ Entrapment efficiency (EE) (table 1). Here, Compritol
888 ATO, Tween 80, and poloxamer 407 were selected as lipid, surfactant, and stabilizers, respectively. Drug concentration (2 $\mathrm{mg} / \mathrm{ml}$ ), HSH speed (10000 rpm) and Poloxamer-407 concentration $(1 \% \mathrm{iw} / \mathrm{w})$, sonication amplitude $(20 \%)$ were set as fix levels.

Using Design Expert software (Version 12.0, Stat-ease. Inc., US), the factorial design was evaluated and a polynomial equation was obtained. In the polynomial equation, the magnitude of the coefficients has positive signs showing a consistent output or a negative sign indicating a contrasting effect. The most appropriate test model (equilibrium, a combination of two, quadratic and cubic model) was determined based on the comparison of statistical parameters such as the coefficient of variation (CV), the coefficient of multiplication $\left(\mathrm{R}^{2}\right)$, the multiple coefficients of correction adjusted ( $\mathrm{R}^{2}$ adjusted), the square residual sum predicted and the 3D response surface plot given by Design-Expert software graphically. The level of significance was considered at a p-value $<0.05$.

Table 1: Selection of variable and their level

\begin{tabular}{lll}
\hline Factor (Independent variable) & Levels & Middle (0) \\
\cline { 2 - 3 } & Low(-1) & High (+1) \\
\hline $\mathrm{X}_{1=}$ Compritol AT0 888 (as a lipid) concentration (\%w/w) & 3 & 5 \\
$\mathrm{X}_{2}=$ Tween 80 (as a Surfactant) concentration (\%w/w) & 1.5 & 2 \\
$\mathrm{X}_{3}=$ HSH Time (min) & 5 & 10 \\
Response (Dependent variable) & Goals & \\
Y1 $=$ Particle size & Minimize & \\
$\mathrm{Y} 2=$ PDI & Minimize & \\
$\mathrm{Y} 3=\%$ iEE & Maximize & \\
\hline
\end{tabular}

\section{Data optimization and model validation}

To create a design space to ensure the quality of the product you want, the impact of each independent CPP on CQA was analyzed. $3^{3}$ factorial design is used in the design space proposal to examine process parameters, the answer to the quality attributes of DOX SLNs is used. The optimization is carried out using an overlay plot (graphical) and desirability (numerical) parameters based on finding the particle size, PDI, and higher EE percentage.

\section{Characterization of optimized DOX-SLNs}

For characterization, optimized DOX-SLNs were formulated and they were characterized for physicochemical, morphological, in vitro drug release, and kinetic studies as shown below:

\section{Compatibility study of lipid and drug}

The compatibility study between the selected lipid and drug was performed by using a Fourier transform-infrared spectrophotometer (FTIR) and Differential scanning calorimetry (DSC).

\section{Fourier transform-infrared spectrophotometer (FTIR)}

FTIR spectroscopy of Doxorubicin hydrochloride, pure lipid excipients, and physical mixture (DOX+lipid) was performed using the Fourier transform-infrared spectrophotometer (Alpha-model Bruker ATR FTIR spectrophotometer). Spectra were scanned at a resolution of $4 \mathrm{~cm}-1$ over a wavelength region of 4000 to $400 \mathrm{~cm}^{-1}$. The process consisted of dispersing $\mathrm{KBr}$ samples and compressing them into discs by applying a hydraulic press pressure of 5 tons for $5 \mathrm{~min}$ ( $\mathrm{KBr}$ pellet method). In the light direction, the pellet was positioned and the spectrum was collected.

\section{Differential scanning calorimetry (DSC)}

DSC measurement of Pure Drug Doxorubicin(DOX), Pure Lipid excipients and Physical mixture (DOX+Lipid), blank (placebo) SLNs, and DOX loaded SLNs formulation was performed with an instrument (Perkin-Elmer Diamond DSC) for measurement of the thermotropic transition of lipids. Empty aluminum pans were used as reference and samples were carefully placed in another aluminum pan. The measurement was done in an inert atmosphere within the temperature range of $30^{\circ} \mathrm{C}$ to $200{ }^{\circ} \mathrm{C}$, at $5^{\circ} \mathrm{C}$ per min.

\section{X-ray diffraction (XRD)}

The crystallographic structure of DOX, Compritol 888 ATO, the physical mixture (drug+lipid), blank SLNs, and DOX-SLNs were characterized by X-ray diffraction (XRD) (D8Advance; Bruker Optik $\mathrm{GmbH}$, Ettlingen, Germany). Samples were exposed to $\mathrm{Cu}-\mathrm{Ka}$ radiation $(40 \mathrm{kV} ; 40 \mathrm{~mA})$ at a scan rate of $0.02 \%$ second over the $2 \theta /$ minute range of $5^{\circ}-50^{\circ}$.

\section{Particle size and polydispersity index (PDI) and zeta potential}

Photon correlation spectroscopy (PCS; Zetasizer, HAS 3000; Malvern Instruments, Malvern, UK)) was performed to determine particle size and Polydispersity Index (PDI). DOX SLNs are diluted 10 times with double distilled water before measuring the size and PDI. Measurements of particle size and PDI were carried out by taking 1 $\mathrm{ml}$ of the diluted formulation into polystyrene cuvettes and disposable folded capillary cells at $25^{\circ} \mathrm{C}$, respectively, for zeta potential. At a wavelength of $633 \mathrm{~nm}$, dynamic light scattering measurements were taken using the helium-neon laser as a light source at a scattering angle of 900 , where particle diffusion is transformed into particle size due to Brownian motion. In the case of zeta potential, particles travel with a velocity related to their zeta potential due to the application of an electric field, which is determined using a technique called phase analysis light scattering and converted to the zeta potential by inbuilt software.

\section{Morphological characterization of optimized DOX SLNs}

Transmission electron microscopy (TEM) (JEM-1200, JEOL Co. Ltd. Tokyo, Japan) was used to visualize the surface morphology of the formulated nanosuspension. A drop of the nanoparticles dispersion was finely spread on a copper grid coated carbon with films and negatively stained with $2 \%(\mathrm{w} / \mathrm{v})$ phosphotungstic acid for viewing [11].

\section{A pH of optimized DOX SLNs}

By taking $10 \mathrm{ml}$ of formulation in a beaker, the $\mathrm{pH}$ of the DOX-loaded SLN was determined. At room temperature, $\mathrm{pH}$ was measured using a calibrated digital $\mathrm{pH}$ meter (Eutech Instruments, $\mathrm{pH}$ tutor, Singapore)

\section{The viscosity of optimized DOX SLNs}

To study the Rheological behavior of the SLNs formulation was determined using Brookfield viscometer (Expert L series, Fungilab Brookfield viscometer). Viscosity determinations were performed at $50 \mathrm{rpm}$ using spindle TL6 at $25 \pm 2{ }^{\circ} \mathrm{C}$.

\section{Entrapment efficiency (\%EE) of DOX SLNs formulated batches}

The percent EE of formulated DOX SLNs was calculated using the method of centrifugation. To obtain lipid nanoparticles, samples were 
collected in centrifuge tubes and centrifuged at $10000 \mathrm{rpm}$ for $20 \mathrm{~min}$ at room temperature. The supernatant was obtained, diluted with methanol appropriately, and analyzed by UV spectroscopy for free drug material. \%EE was calculated by following equation (1): [12]

$$
\% \mathrm{EE}=\frac{\text { Total amount of DOX }- \text { Amount of free DOX }}{\text { The total amount of DOX }} \ldots \ldots \ldots \text {..... eq (1) }
$$

\section{In vitro drug release of optimized DOX SLNs and release kinetics} mechanism

Using a dialysis bag diffusion technique, in vitro drug release was assessed. A total of $5 \mathrm{ml}$ of the sample was taken into a dialysis bag and submerged in a beaker containing $200 \mathrm{ml}$ of phosphate-buffered saline $(\mathrm{pH} 7.4)$ [42] at $37 \pm 1{ }^{\circ} \mathrm{C}$ for $12 \mathrm{~h}$ with continuous stirring using a shaking incubator at a speed of $100 \mathrm{rpm}$ (REMI Instruments Ltd). An aliquot of $1 \mathrm{ml}$ of dissolving medium was removed at a fixed interval and the same volume of fresh medium was applied. Then the absorption of withdrawn samples was measured at $480 \mathrm{~nm}$ against a blank reagent using the UV spectrophotometer (Agilent Cary $60 \mathrm{UV}$-Vis spectrophotometer). Throughout the experiment, sink conditions were preserved.

All the experiments were performed in triplicate. The data obtained from the in vitro drug release analysis were adapted to various kinetic models viz., zero-order (cumulative drug release percentage versus time), first-order (cumulative drug release log remaining versus time), Higuchi model (cumulative drug release percentage versus square root of time) Hixon Crowell model (cumulative drug root remaining versus time) and Korsmeyer-Peppas model (Log percent CDR versus log time) [13]

\section{Stability studies}

The stability analysis aimed to determine the effect of the presence of formulation additives on the stability of the drug and also to determine the physical stability of the formulation prepared under storage and relative humidity conditions. Stability tests at $4 \pm 2{ }^{\circ} \mathrm{C}$ (refrigerator), $25 \pm 2{ }^{\circ} \mathrm{C} / 60 \pm 5$ percent $\mathrm{RH}$ and $40 \pm 2{ }^{\circ} \mathrm{C} / 75 \pm 5$ percent $\mathrm{RH}$ in the stability chamber (Oswald Scientific Equipments Pvt. Ltd, Mumbai India) were carried out on optimized DOX-SLNs. After 0, 1 , 3 , and $6 \mathrm{mo}$, the sample was extracted and the effects on particle size, shape, PDI, zeta potential, and entrapment efficiency were determined.

\section{RESULTS AND DISCUSSION}

\section{The solubility of DOX in lipids}

A limiting factor in the formulation of SLNs is the amount of solid lipid needed to solubilize the drug. In Solid lipid nanosuspension formulation, the amount of lipid required to solubilize the drug is a crucial factor as it has a significant impact on the \% entrapment efficiency. As per the details shown in fig. 1, to conduct the solubility analysis, five distinct solid lipids were taken. DOX solubility in Compritol 888 ATO required a lower quantity, suggesting higher drug solubility. As it has been successfully used in various pharmaceutical dosage types, Compritol 888 ATO has attracted particular interest. The applicability of Compritol 888 ATO in the preparation of aqueous colloidal dispersions such as solid lipid microparticles (SLMs), solid lipid nanoparticles (SLNs) and nanostructured lipid carriers (NLCs) to capture lipophilic drugs has been highlighted in various research studies [14-20].
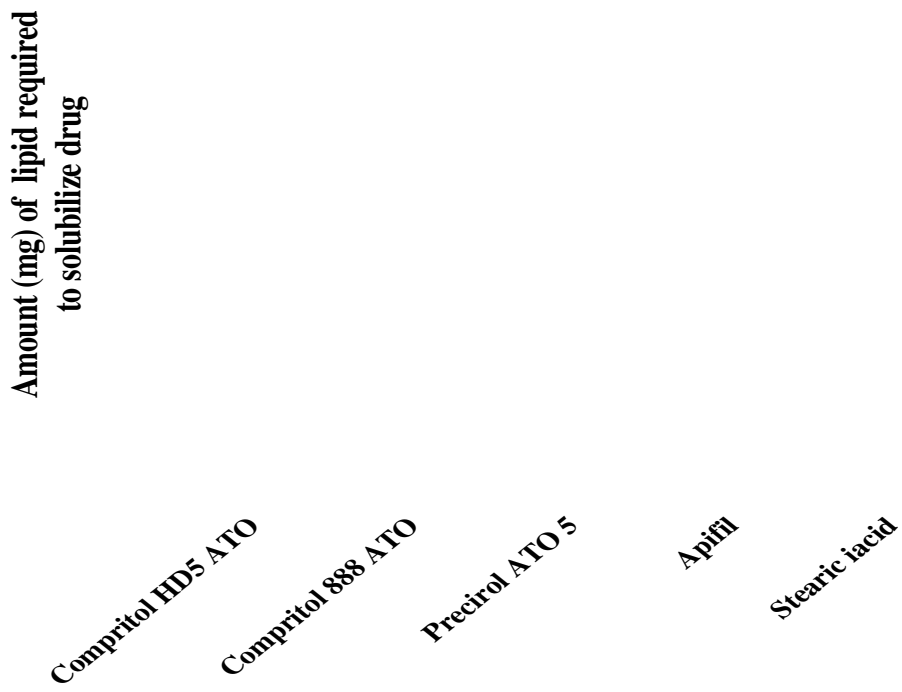

Fig. 1: Comparison of the amount of lipid required to solubilized DOX

\section{$3^{3}$ factorial design}

A significant and critical problem in the production of pharmaceutical dosage forms is the investigation of an ideal formula for the design of medication of the desired quality through minimum trials within a short period. In the literature on the development of different pharmaceutical dosage types, optimization through computer-aided statistical experimental design methodologies has been successfully applied [21]. The technique of statistical optimization involves designing a series of experiments that will calculate the response variables accurately, fitting the data with mathematical models, performing sufficient statistical tests to choose the best possible model, and producing an optimal response; the values of independent formulation variables are calculated. A factorial design is one of the common experimental designs used in the optimization of formulations between different statistical designs [22].

\section{Selection of factors, levels, and Responses of experimental design}

$3^{3}$ factorial was developed to analyze the main effect, the interaction effect, and the quadratic effect of three independent variables (CPP) on the three responses (CQA). In this case, 30 experiments run were performed, and the results were shown in table 2 . 
Table 2: Observed response for size, PDI, and \% EE of factorial runs

\begin{tabular}{|c|c|c|c|c|c|c|}
\hline Run & X1 & X2 & X3 & Y1 & Y2 & Y3 \\
\hline VS1 & 3 & 1.5 & 5 & $252.5 \pm 5.29$ & $0.32 \pm 0.074$ & $52.1 \pm 2.13$ \\
\hline VS2 & 4 & 1.5 & 5 & $239.1 \pm 4.87$ & $0.291 \pm 0.022$ & $58.5 \pm 1.89$ \\
\hline VS 3 & 5 & 1.5 & 5 & $279.7 \pm 3.43$ & $0.327 \pm 0.03$ & $62 \pm 1.11$ \\
\hline VS 4 & 3 & 2 & 5 & $218.9 \pm 6.55$ & $0.306 \pm 0.051$ & $54.6 \pm 2.56$ \\
\hline VS 5 & 4 & 2 & 5 & $200.6 \pm 4.11$ & $0.257 \pm 0.029$ & $64 \pm 2.44$ \\
\hline VS 6 & 5 & 2 & 5 & $239.2 \pm 5.01$ & $0.325 \pm 0.04$ & $71.7 \pm 1.21$ \\
\hline VS 7 & 3 & 2.5 & 5 & $279.2 \pm 3.72$ & $0.361 \pm 0.036$ & $47.1 \pm 1.35$ \\
\hline VS 8 & 4 & 2.5 & 5 & $285.3 \pm 4.36$ & $0.35 \pm 0.064$ & $51.2 \pm 2.47$ \\
\hline VS 9 & 5 & 2.5 & 5 & $306.8 \pm 5.63$ & $0.393 \pm 0.02$ & $61.6 \pm 3.12$ \\
\hline VS 10 & 3 & 1.5 & 10 & $188.2 \pm 3.08$ & $0.235 \pm 0.05$ & $57.4 \pm 2.40$ \\
\hline VS 11 & 4 & 1.5 & 10 & $165.3 \pm 3.51$ & $0.211 \pm 0.047$ & $67.2 \pm 1.39$ \\
\hline VS 12 & 5 & 1.5 & 10 & $228.5 \pm 4.13$ & $0.27 \pm 0.059$ & $73.1 \pm 2.78$ \\
\hline VS 13 & 3 & 2 & 10 & $174.4 \pm 5.29$ & $0.25 \pm 0.067$ & $65.2 \pm 3.29$ \\
\hline VS 14 & 4 & 2 & 10 & $142.5 \pm 2.05$ & $0.201 \pm 0.018$ & $74.2 \pm 2.87$ \\
\hline VS 15 & 5 & 2 & 10 & $199.4 \pm 1.33$ & $0.278 \pm 0.083$ & $79 \pm 1.55$ \\
\hline VS 16 & 3 & 2.5 & 10 & $208.2 \pm 2.58$ & $0.307 \pm 0.077$ & $52.7 \pm 2.31$ \\
\hline VS 17 & 4 & 2.5 & 10 & $222.1 \pm 4.77$ & $0.318 \pm 0.045$ & $58.7 \pm 3.33$ \\
\hline VS 18 & 5 & 2.5 & 10 & $239.6 \pm 6.63$ & $0.299 \pm 0.089$ & $67.5 \pm 3.59$ \\
\hline VS 19 & 3 & 1.5 & 15 & $196.1 \pm 5.97$ & $0.423 \pm 0.049$ & $43.1 \pm 3.54$ \\
\hline VS 20 & 4 & 1.5 & 15 & $183.9 \pm 6.28$ & $0.401 \pm 0.09$ & $49.4 \pm 2.65$ \\
\hline VS 21 & 5 & 1.5 & 15 & $246.7 \pm 8.39$ & $0.451 \pm 0.057$ & $59.8 \pm 2.48$ \\
\hline VS 22 & 3 & 2 & 15 & $191.2 \pm 3.99$ & $0.385 \pm 0.03$ & $45.2 \pm 1.66$ \\
\hline VS 23 & 4 & 2 & 15 & $171.4 \pm 9.25$ & $0.36 \pm 0.063$ & $53.5 \pm 2.09$ \\
\hline VS 24 & 5 & 2 & 15 & $225 \pm 4.58$ & $0.40 \pm 0.087$ & $62 \pm 2.98$ \\
\hline VS 25 & 3 & 2.5 & 15 & $248.7 \pm 6.89$ & $0.441 \pm 0.091$ & $39.8 \pm 3.23$ \\
\hline VS 26 & 4 & 2.5 & 15 & $261.2 \pm 7.15$ & $0.465 \pm 0.05$ & $47.2 \pm 2.29$ \\
\hline VS 27 & 5 & 2.5 & 15 & $251.1 \pm 4.92$ & $0.446 \pm 0.028$ & $55.4 \pm 1.73$ \\
\hline VS 28 & 4 & 2 & 10 & $137.3 \pm 7.50$ & $0.187 \pm 0.07$ & $77.2 \pm 1.19$ \\
\hline VS 29 & 4 & 2 & 10 & $148 \pm 6.25$ & $0.20 \pm 0.062$ & $73.6 \pm 1.47$ \\
\hline VS30 & 4 & 2 & 10 & $143.2 \pm 4.10$ & $0.208 \pm 0.07$ & $71.7 \pm 1.56$ \\
\hline
\end{tabular}

Data represent mean $\pm \mathrm{SD}, \mathrm{n}=3$

A quadratic model was fitted into the data of experimental results by the use of Design-Expert® Software Version 12.

The following main criteria are fitted according to the best model:

1. High $F$-value,

2. Low $P$-value $(<0.05)$,

3. Insignificant lack of fit,
4. High $R 2(>0.90)$

5. Low standard deviation,

6. A randomly scatter plot of residuals, and

7. Whether it can predict well the validation set.

Data have shown in table 3 indicated that the p-value for all three CQA was $>0.05$ for cubic and quadratic models.

Table 3: Model fit summary of responses

\begin{tabular}{|c|c|c|c|c|c|c|}
\hline Response & Source & Sequential p-value & Lack of fit p-value & Adjusted R ${ }^{2}$ & Predicted $\mathbf{R}^{2}$ & Remarks \\
\hline \multirow[t]{4}{*}{ Size } & Linear & 0.0513 & 0.0014 & 0.1568 & 0.0301 & \\
\hline & $2 \mathrm{FI}$ & 0.9424 & 0.0012 & 0.0699 & -0.3776 & \\
\hline & Quadratic & $<0.0001$ & 0.0644 & 0.9833 & 0.9339 & Suggested \\
\hline & Cubic & 0.3157 & 0.0255 & 0.8950 & 0.7147 & Aliased \\
\hline \multirow[t]{4}{*}{ PDI } & Linear & 0.0371 & 0.0019 & 0.1779 & 0.0484 & \\
\hline & $2 \mathrm{FI}$ & 0.9056 & 0.0016 & 0.0992 & -0.3201 & \\
\hline & Quadratic & $<0.0001$ & 0.0704 & 0.9323 & 0.9095 & Suggested \\
\hline & Cubic & 0.3165 & 0.0740 & 0.9391 & 0.8376 & Aliased \\
\hline \multirow[t]{4}{*}{$\% \mathrm{EE}$} & Linear & 0.0024 & 0.0215 & 0.3329 & 0.2442 & \\
\hline & $2 \mathrm{FI}$ & 0.9602 & 0.0182 & 0.2616 & -0.0022 & \\
\hline & Quadratic & $<0.0001$ & 0.4174 & 0.9324 & 0.9035 & Suggested \\
\hline & Cubic & 0.8028 & 0.3255 & 0.9190 & 0.7444 & Aliased \\
\hline
\end{tabular}

Warning: The Cubic model is aliased. Quadratic models were found to be best fit models for all dependent variables viz., size, PDI, and \%EE, and the $\mathrm{R}^{2}$ values for the quadratic model were found to be $0.9339,0.9095$, and 0.9035 whereas for the cubic model R2 values were $0.7147,0.8376$ and 0.7444 respectively. These models were evaluated statistically by applying one-way ANOVA (p<0.05). The result of the ANOVA is shown in table 4

\section{Effect of the independent variable on particle size}

The particle size of factorial runs varied between 137.3 to $306.8 \mathrm{~nm}$ as shown in the counterplot and 3D response plot in fig. 2 and 3 .

The polynomial equations for size

Size $=+145.46+13.90 \mathrm{~A}+16.97 \mathrm{~B}-18.57 \mathrm{C}-6.96 \mathrm{AB}$ $0.1444 A C+0.6196 B C+28.53 A^{2}+39.95 B^{2}+45.73 C^{2}---------e q u a t i o n-2$

Where $A, B, C, A B, A C, B C, A^{2}, B^{2}, C^{2}$ in Equation (2) were significant model terms.
As shown in equation 2, Positive coefficients of factor $\mathrm{A}, \mathrm{B}, \mathrm{AC}, \mathrm{BC}$, $A^{2}, B^{2}, C^{2}$ denotes synergistic effect on particle size, while negative coefficients of $\mathrm{C}, \mathrm{AB}$ denotes antagonistic effect on particle size. The Predicted $\mathrm{R}^{2}$ of 0.9339 was in reasonable agreement with the adjusted R2 of 0.9833 , indicating the adequacy of the model to predict the response of particle size.

\section{Effect of lipid concentration on particle size}

It was observed that Conc. of Compritol 888 ATO (lipid concentration) had a positive effect on particle size (Y1). It was also 
observed that as lipid concentration increased, the dispersed phase viscosity also increased, resulting in higher-size particle agglomeration and reduced homogenization ability.

\section{Effect of surfactant concentration on particle size}

As per results shown in table 2 for various experimental runs, as the concentration of tween 80 (Surfactant concentration) increased from $1.5 \%$ to $2.5 \% \mathrm{w} / \mathrm{w}$, the size was decreasing and then with 2.5, it is increased. With increasing concentration of tween 80 from $1.5 \%$ to $2.5 \% \mathrm{w} / \mathrm{w}$, it showed reduced interfacial tension between lipid and aqueous phase, which may control the aggregation of lipid particle by facilitating the particle partition thereby resulting in lower size [23]. Higher surfactant concentrations, as recorded in previous studies, effectively stabilize the lipid matrix by forming a steric barrier on its surface, thereby preventing aggregation [24]. When $2.5 \%$ $\mathrm{w} / \mathrm{w}$ tween 80 was used, the size was constantly increasing, it was observed. This was because the alkyl chain of the surfactant molecule covers the lipid particle surface through hydrophobic interaction to form a stable lipid matrix during the homogenization process. Once this stable matrix has been formed, an excess surfactant can cause surfactant particles to accumulate on the surface of the stable lipid matrix, causing an increase in size in our case [25].

\section{Effect of HSH time on particle size}

Homogenization was performed at 10000 RPM for three different time intervals viz., 5, 10, and 15 min. with an increase in HT from 5 to $10 \mathrm{~min}$, the size was gradually decreasing, while at $15 \mathrm{~min}$ size was increasing. One of the important techniques for applying kinetic energy to achieve a lower size is the speed and time of homogenization for which it is used. Applying high kinetic energy for longer periods can lead to instability of the lipid structures produced, resulting in larger particles being aggregated and formed. In contrast to $10 \mathrm{~min}$ of $\mathrm{HT}$, the size was higher at $5 \mathrm{~min}$ due to inadequate homogenization. An optimum homogenization time would therefore contribute to the creation of stable particles with a uniform distribution of size.

Table 4: Anova response size, PDI, and EE

\begin{tabular}{|c|c|c|c|c|c|c|}
\hline Response 1: size (nm) & SS & df & MS & F-value & p-value & Remarks \\
\hline Model & 58875.49 & 9 & 6541.72 & 27.06 & $<0.0001$ & significant \\
\hline A-Conc. of Compritol 888 ATO & 3564.37 & 1 & 3564.37 & 14.74 & 0.0009 & \\
\hline B-Conc. of Tween 80 & 5731.70 & 1 & 5731.70 & 23.71 & $<0.0001$ & \\
\hline C-HSH Time & 6361.58 & 1 & 6361.58 & 26.32 & $<0.0001$ & \\
\hline$A B$ & 601.33 & 1 & 601.33 & 2.49 & 0.1290 & \\
\hline $\mathrm{AC}$ & 0.2522 & 1 & 0.2522 & 0.0010 & 0.9745 & \\
\hline $\mathrm{BC}$ & 4.76 & 1 & 4.76 & 0.0197 & 0.8897 & \\
\hline$A^{2}$ & 5746.86 & 1 & 5746.86 & 23.77 & $<0.0001$ & \\
\hline $\mathrm{B}^{2}$ & 11596.89 & 1 & 11596.89 & 47.97 & $<0.0001$ & \\
\hline $\mathrm{C}^{2}$ & 14764.97 & 1 & 14764.97 & 61.08 & $<0.0001$ & \\
\hline Residual & 5318.17 & 22 & 241.73 & & & \\
\hline Lack of Fit & 5260.64 & 19 & 276.88 & 14.44 & 0.0644 & not significant \\
\hline Pure Error & 57.53 & 3 & 19.18 & & & \\
\hline Cor Total & 64193.66 & 31 & & & & \\
\hline Response: 2 PDI & SS & $\mathrm{df}$ & MS & F-value & $\mathrm{p}$ value & Remarks \\
\hline Model & 0.1996 & 9 & 0.0222 & 48.46 & $<0.0001$ & significant \\
\hline A-Conc. of Compritol 888 ATO & 0.0012 & 1 & 0.0012 & 2.72 & 0.1134 & \\
\hline B-Conc. of Tween 80 & 0.0106 & 1 & 0.0106 & 23.07 & $<0.0001$ & \\
\hline C-HSH Time & 0.0391 & 1 & 0.0391 & 85.44 & $<0.0001$ & \\
\hline $\mathrm{AB}$ & 0.0005 & 1 & 0.0005 & 1.10 & 0.3064 & \\
\hline $\mathrm{AC}$ & 0.0001 & 1 & 0.0001 & 0.1959 & 0.6623 & \\
\hline $\mathrm{BC}$ & 0.0013 & 1 & 0.0013 & 2.91 & 0.1022 & \\
\hline$A^{2}$ & 0.0080 & 1 & 0.0080 & 17.50 & 0.0004 & \\
\hline $\mathrm{B}^{2}$ & 0.0132 & 1 & 0.0132 & 28.93 & $<0.0001$ & \\
\hline $\mathrm{C}^{2}$ & 0.0926 & 1 & 0.0926 & 202.33 & $<0.0001$ & \\
\hline Residual & 0.0101 & 22 & 0.0005 & & & \\
\hline Lack of Fit & 0.0098 & 19 & 0.0005 & 6.75 & 0.0704 & not significant \\
\hline Pure Error & 0.0002 & 3 & 0.0001 & & & \\
\hline Cor total & 0.2096 & 31 & & & & \\
\hline Response: 3 EE \% & SS & df & MS & F-value & $\mathrm{p}$ value & Remarks \\
\hline Model & 3248.97 & 9 & 361.00 & 48.49 & $<0.0001$ & significant \\
\hline A-Conc. of Compritol 888 ATO & 1066.48 & 1 & 1066.48 & 143.25 & $<0.0001$ & \\
\hline B-Conc. of Tween 80 & 85.95 & 1 & 85.95 & 11.55 & 0.0026 & \\
\hline C-HSH Time & 243.47 & 1 & 243.47 & 32.70 & $<0.0001$ & \\
\hline$A B$ & 4.33 & 1 & 4.33 & 0.5810 & 0.4540 & \\
\hline $\mathrm{AC}$ & 8.14 & 1 & 8.14 & 1.09 & 0.3071 & \\
\hline $\mathrm{BC}$ & 4.57 & 1 & 4.57 & 0.6142 & 0.4416 & \\
\hline$A^{2}$ & 6.61 & 1 & 6.61 & 0.8876 & 0.3564 & \\
\hline $\mathrm{B}^{2}$ & 401.08 & 1 & 401.08 & 53.87 & $<0.0001$ & \\
\hline $\mathrm{C}^{2}$ & 1159.94 & 1 & 1159.94 & 155.81 & $<0.0001$ & \\
\hline Residual & 163.79 & 22 & 7.44 & & & \\
\hline Lack of Fit & 148.18 & 19 & 7.80 & 1.50 & 0.4174 & not significant \\
\hline Pure Error & 15.61 & 3 & 5.20 & & & \\
\hline Cor Total & 3412.75 & 31 & & & & \\
\hline
\end{tabular}

SS= Sum of squares; $\mathrm{df}=$ degree of freedom; MS= Mean square, The probability value (p-value) by the regression analysis is shown in table 3 and the $\mathrm{F}$ test value is shown in table 4 for the three CQAs with a confidence level of $95 \%$. The large p-value for lack of fit $(>0.05)$ indicates that the lack of fit test is insignificant, implying that a significant model correlation existed between the CPP and CQA. 
Table 5: Lack of Fit Tests particle size, PDI, and \%EE

\begin{tabular}{|c|c|c|c|c|c|c|c|}
\hline Response & Source & Sum of squares & df & Mean square & F-value & p-value & Remarks \\
\hline \multirow[t]{5}{*}{ size } & Linear & 48832.15 & 25 & 1953.29 & 101.86 & 0.0014 & \\
\hline & $2 \mathrm{FI}$ & 48090.42 & 22 & 2185.93 & 113.99 & 0.0012 & \\
\hline & Quadratic & 5260.64 & 19 & 276.88 & 14.44 & 0.0644 & Suggested \\
\hline & Cubic & 2986.52 & 11 & 271.50 & 14.16 & 0.0255 & Aliased \\
\hline & Pure Error & 57.53 & 3 & 19.18 & & & \\
\hline \multirow[t]{5}{*}{ PDI } & Linear & 0.1554 & 25 & 0.0062 & 81.10 & 0.0019 & \\
\hline & $2 \mathrm{FI}$ & 0.1521 & 22 & 0.0069 & 90.15 & 0.0016 & \\
\hline & Quadratic & 0.0098 & 19 & 0.0005 & 6.75 & 0.0704 & Suggested \\
\hline & Cubic & 0.0055 & 11 & 0.0005 & 6.56 & 0.0740 & Aliased \\
\hline & Pure Error & 0.0002 & 3 & 0.0001 & & & \\
\hline \multirow[t]{5}{*}{$\% \mathrm{EE}$} & Linear & 2040.60 & 25 & 81.62 & 15.69 & 0.0215 & \\
\hline & $2 \mathrm{FI}$ & 2016.59 & 22 & 91.66 & 17.62 & 0.0182 & \\
\hline & Quadratic & 148.18 & 19 & 7.80 & 1.50 & 0.4174 & Suggested \\
\hline & Cubic & 109.17 & 11 & 9.92 & 1.91 & 0.3255 & Aliased \\
\hline & Pure Error & 15.61 & 3 & 5.20 & & & \\
\hline
\end{tabular}

*The selected model should have an insignificant lack-of-fit.

\section{Effect of the independent variable on PDI}

As shown in the counterplot and 3D response plot in fig. 2 and 3, the PDI of factorial runs ranged from 0.187 to 0.465

The polynomial equations for PDI were as per following;

PDI $=+0.2057+0.0082 \mathrm{~A}+0.0230 \mathrm{~B}+0.0460 \mathrm{C}-0.0064 \mathrm{AB}-0.0027 \mathrm{AC}-$ $0.0104 \mathrm{BC}+0.0337 \mathrm{~A}^{2}+0.0427 \mathrm{~B}^{2}+0.1145 \mathrm{C}^{2}----------$ equation-3

Where A, B, C, AB, AC, BC, $A^{2}, B^{2}, C^{2}$ in Equation (3) were significant model terms.

Positive coefficients of factors $A, B, C, A^{2}, B^{2}, C^{2}$ denote synergistic effect on PDI as shown in equation 3 , whereas negative coefficients of factor $\mathrm{AB}, \mathrm{BC}, \mathrm{AC}$ denote antagonistic effect on PDI. The predicted R2 0.9095 was in good agreement with the modified $\mathrm{R}^{2}$ 0.9323, indicating the adequacy of the model for predicting PDI.

In terms of actual variables, the equation can be used to make predictions of the answer for each factor at given levels. Here, for each factor, levels should be defined in the original units.

To calculate the relative impact of each factor, this equation should not be used because the coefficients are scaled to fit the units of each factor and the intercept is not at the center of the design space.

\section{Effect of lipid concentration on PDI}

It was observed that Conc. of Compritol 888 ATO (lipid concentration) had a positive effect on PDI (Y2). It was also observed that as lipid concentration increased, the dispersed phase viscosity also increased, resulting in greater PDI due to particle agglomeration and reduced homogenization ability.

\section{Effect of surfactant concentration on PDI}

As per the results shown in table 2 for different experimental runs, PDI decreased as the concentration of tween 80 (surfactant concentration) increased from $1.5 \%$ to $2.5 \% \mathrm{w} / \mathrm{w}$. With an increased concentration of tween 80 from 1.5 to $2.5 \% \mathrm{w} / \mathrm{w}$, decreased interfacial tension between lipid and aqueous phase was shown to regulate lipid particle aggregation by facilitating particle partition, resulting in lower PDI [23]. It was observed that PDI increased continuously when $2.5 \% \mathrm{w} / \mathrm{w}$ tween 80 was used,

\section{Effect of HSH time on PDI}

For three separate time intervals, viz., 5, 10, and 15 min, homogenization was performed at 10000 RPM. PDI decreased gradually with an increase in HT from 5 to $10 \mathrm{~min}$, while PDI increased gradually at $15 \mathrm{~min}$.

\section{Effect of the independent variable on \% entrapment efficiency} (EE \%)

As shown in the counterplot and 3D response plot in fig. 2 and 3, the $\%$ EE of factorial runs ranged from 39.8 to 79.
The polynomial equations for $\% \mathrm{EE}$ were as per following;

$\%$ Entrapment Efficiency (EE \%)=+72.88+7.61A-2.08B-3.63C+ $0.5906 \mathrm{AB}+0.8203 \mathrm{AC}+0.6073 \mathrm{BC}-0.9674 \mathrm{~A}^{2}-7.43 \mathrm{~B}^{2}-12.82 \mathrm{C}^{2}-\ldots-{ }_{-}-{ }_{-}$ equation 4

Where $\mathrm{A}, \mathrm{B}, \mathrm{C}, \mathrm{AB}, \mathrm{AC}, \mathrm{BC}, \mathrm{A}^{2}, \mathrm{~B}^{2}, \mathrm{C}^{2}$ in Equation (4) were significant model terms.

As shown in equation 4 , the positive factor $\mathrm{A}, \mathrm{AB}, \mathrm{BC}, \mathrm{AC}$ coefficients denote a synergistic effect on percent $\mathrm{EE}$, while the negative factor $\mathrm{B}$, $C, A^{2}, B^{2}, C^{2}$ coefficients denote an antagonistic effect on percent EE. The predicted $\mathrm{R}^{2} 0.9035$ was in good agreement with the modified $\mathrm{R}^{2} 0.9324$ suggested the adequacy of the model for forecasting the $\%$ EE response.

\section{Effect of lipid concentration on \% EE}

It was observed that Conc. of Compritol 888 ATO (lipid concentration) had a positive effect on $\%$ EE (Y3). It was also observed that as lipid concentration increased, the viscosity of the dispersed phase also increased, resulting in decreased homogenization efficiency of particle agglomeration. The higher \% EE may be due to the presence of higher lipid concentrations, which provide additional space for the drug molecule to be embedded, thus reducing the overall surface area. As the viscosity of the lipid phase is higher and thus shows higher \%EE [26, 27], this can lead to a reduction in the diffusion rate of the solute molecule. From the outcome shown in table 4 , it was observed that $3 \%$ lipid concentration showed weak \% EE of $39.8 \pm 3.23$ compared to $5 \%$ lipid concentration showing more than $55 \% \mathrm{EE}$ in all experimental runs because lipid quantity was higher.

\section{Effect of surfactant concentration on \% EE}

As shown in table 2 for different experimental runs, as the concentration of tween 80 (surfactant concentration) increased from $1.5 \%$ to $2.0 \% \mathrm{w} / \mathrm{w}$, EE percent increased. It was found that the percent $\mathrm{EE}$ was reduced when 2.5 percent $\mathrm{w} / \mathrm{w}$ tween 80 was used. The reduction in EE percent could be due to a higher solubilization effect produced by a higher DOX surfactant concentration. DOX solubility in the external phase can increase at higher surfactant concentrations due to the diffusion of the drug from the lipid core into the aqueous phase, resulting in a decreased \% EE. The findings were consistent with the results of another group describing a lower EE percentage, which may be attributed to the solubilization effect of the emulsifier on the aqueous phase drug molecule [27].

\section{Effect of HSH time on \% EE}

The \% EE was found to be lower in 15 min than in 10 min. This was attributable to the removal from the lipid surface of surfactant particles, which induced lipid disruption and the escape of the trapped drug into the aqueous process. 

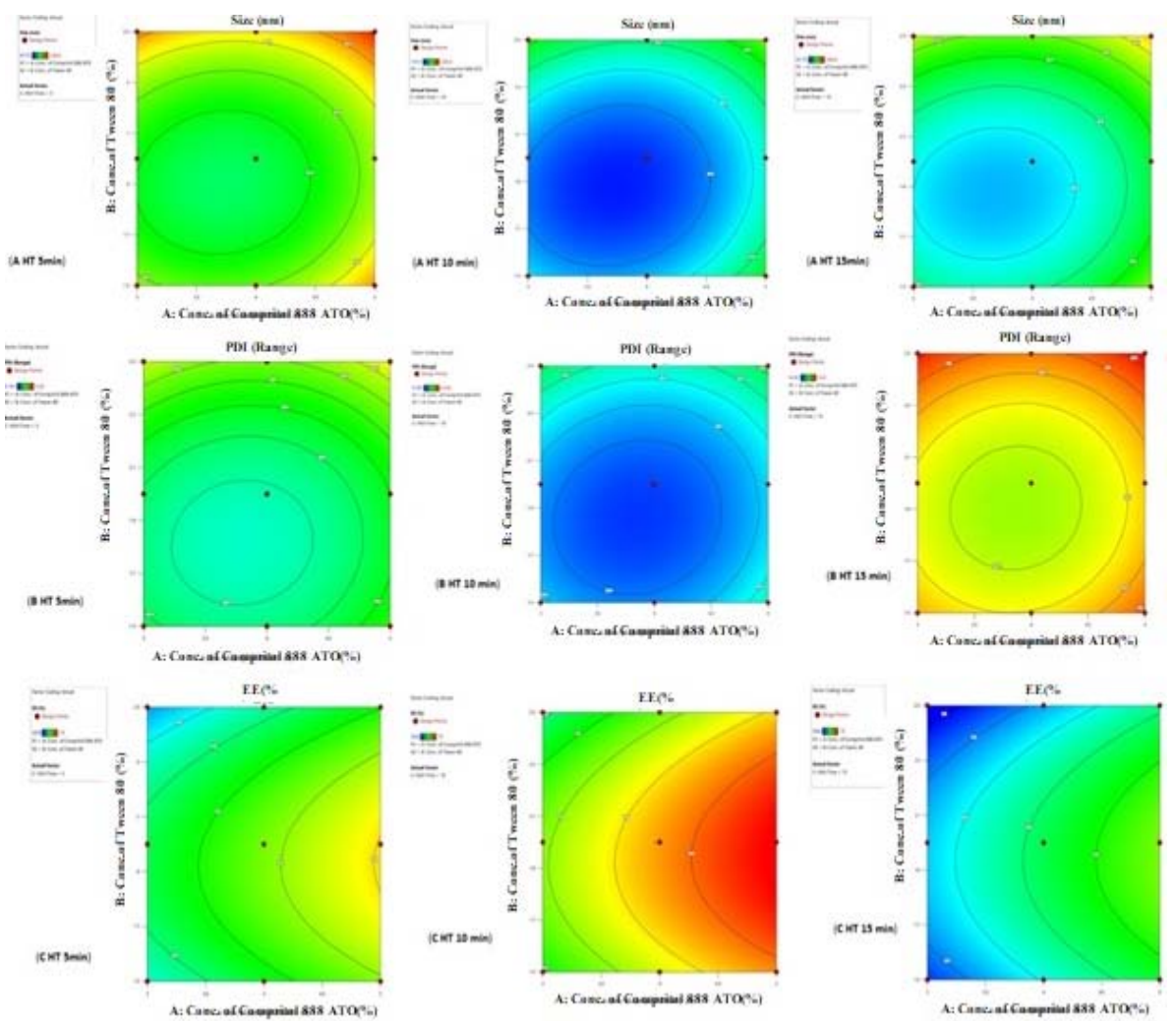

Fig. 2: Counter plot with the effect of formulation and process variable on A) Particle size B) PDI C) \%EE

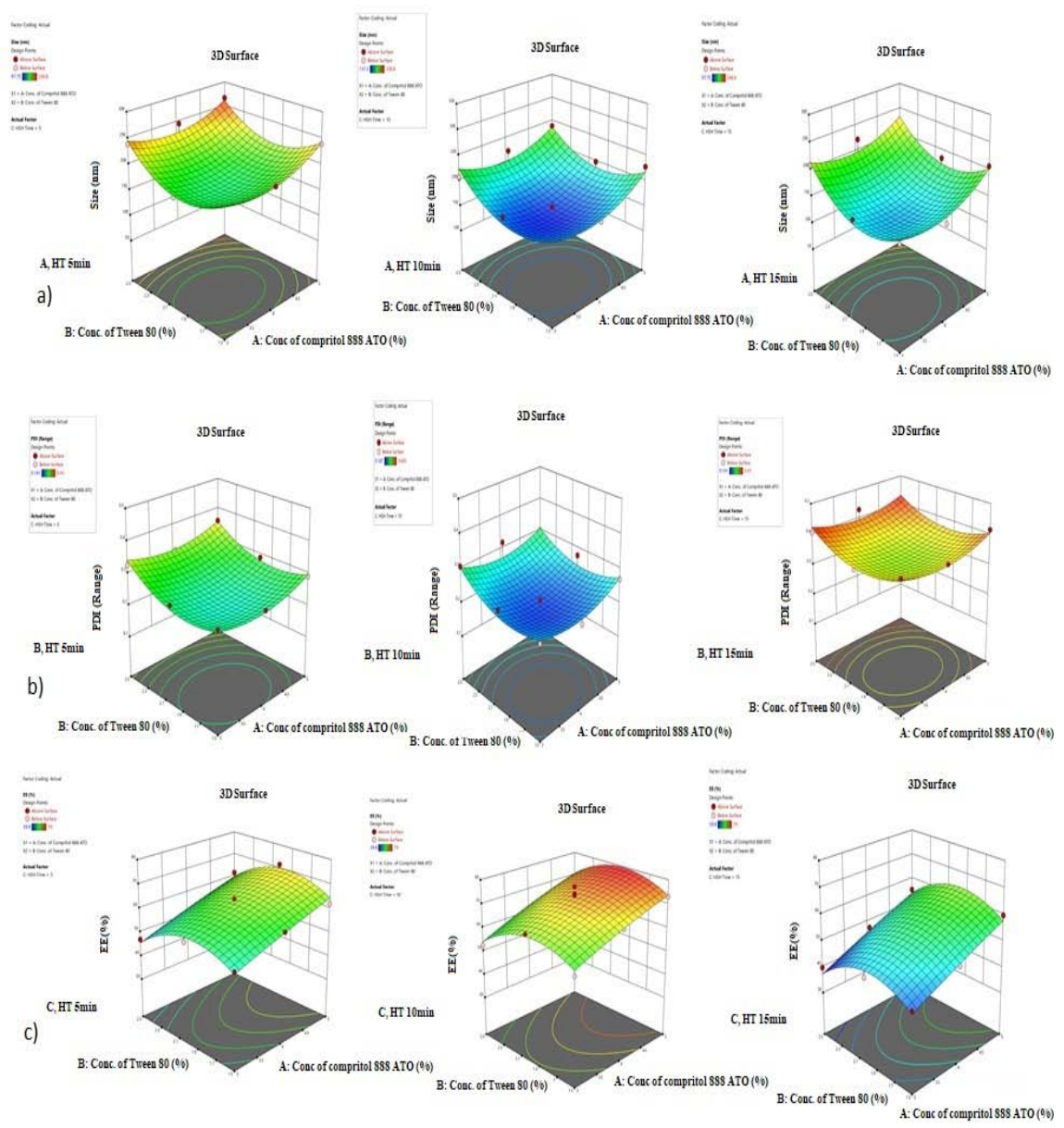

Fig. 3: 3D response surface plot with the effect of formulation and process variable on A) Particle size B) PDI C) \%EE 
Optimization of data and validation of response surface methodology

To create new formulations with the desired response (optimum quality), a numerical optimization method using the desirability approach was used. The desired ranges of in dependable variables (factors) were limited to $3 \leq \mathrm{A} \leq 5 \% 1.5 \leq \mathrm{B} \leq 2.5 \%$, and $5 \leq \mathrm{C} \leq 15 \mathrm{~min}$, while the desired response ranges were limited to $200 \leq \mathrm{Y} 1 \leq 100 \mathrm{~nm}$, $0.15 \leq Y 2 \leq 0.3$, and $70 \leq Y 3 \leq 80 \%$. The optimum response values were obtained by numerical analysis using the Design-Expert 12 software and one of them was chosen based on the desirability criterion.

Optimized DOX loaded Solid Lipid-based Nanosuspension (SLNs) optimized process variable settings proposed by design were prepared using the homogenization and ultrasonication to evaluate the optimization capability of the mathematical models produced according to the results of the complete $3^{3}$ factorial design.

By formulating and characterizing nanoparticles at the checkpoint batch suggested by the software, experimental validation of DoE trials for formulation variables was carried out. The overlay plot showing the design space and configured parameters as a checkpoint proposed by DoE software to obtain the desired responses is shown in fig. 4.

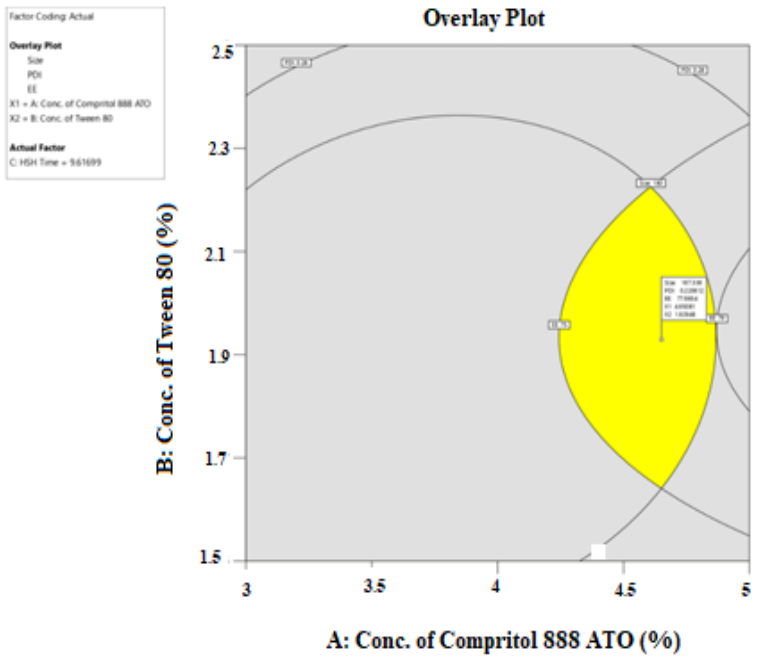

Fig. 4: The overlay plot indicating the region of optimal process variable settings

Table 6: Result of an experiment for confirming optimization capability

\begin{tabular}{llll}
\hline Code & Factor & & \\
\cline { 2 - 4 } & Conc. of compritol 888 ATO A (\%) & Conc. of tween 80 B (\%) & HSH time C (Min) \\
\hline V-O & 4.6 & 1.9 & 10 \\
Responses & Predicted value & a Observed Value & besidual \\
$Y_{1}=$ Particle size (nm) & 167.339 & $167.47 \pm 6.09$ & -0.12 \\
$Y_{2}=$ PDI & 0.220616 & $0.23 \pm 0.02$ & 0.01 \\
$Y_{3}=$ Entrapment efficiency $(\%)$ & 77.6838 & $75.3 \pm 2.79$ & 2.38 \\
\hline
\end{tabular}

aObserved response values: mean \pm SD $(n=3)$., bResidual= [difference between predicted value and actual value], The chosen optimal process variable setting used for the optimized formulation was Conc. Compritol 888 ATO $(\mathrm{A})=4.6 \%$, Conc. Tween $80(\mathrm{~B})=1.9 \%$ and time $(\mathrm{C})$ of HSH $=10$ min. Optimized DOX-Loaded lipid-based Nano suspension (V-0) formulation was evaluated for response Y1: Particle size (nm), Y2: PDI, and Y3: Entrapment efficiency (\%).

Table 7: Point prediction confirmation table

\begin{tabular}{|c|c|c|c|c|c|c|c|c|c|}
\hline $\begin{array}{l}\text { Solution } 1 \text { of } \\
1 \text { response }\end{array}$ & $\begin{array}{l}\text { Predicted } \\
\text { mean }\end{array}$ & $\begin{array}{l}\text { Predicted } \\
\text { median }\end{array}$ & Observed & Std Dev & n & SE pred & 95\% PI low & $\begin{array}{l}\text { Data } \\
\text { mean }\end{array}$ & 95\% PI high \\
\hline Size & 167.339 & 167.339 & 167.47 & 15.5478 & 3.00 & 10.69 & 145.153 & 167.47 & 189.525 \\
\hline PDI & 0.220616 & 0.220616 & 0.23 & 0.0214 & 3.00 & 0.0147 & 0.190091 & 0.23 & 0.25114 \\
\hline EE & 77.6838 & 77.6838 & 75.3 & 2.72851 & 3.00 & 1.877 & 73.7904 & 75.3 & 81.5773 \\
\hline
\end{tabular}

*Interval: Two-sided, Confidence $=95 \%$, alpha $=0.05$, The observed values (particle size $167.47 \pm 6.09 \mathrm{~nm}$, PDI $0.23 \pm 0.02$, and EE $75.3 \pm 2.79 \%$ ) were comparable to the expected values (particle size 167.33. nm, PDI 0.22, and EE 77.68\%) to assess the optimization procedure's reliability.

\section{Characterization of optimized DOX-SLNs formulation}

\section{Fourier transform infrared (FTIR) spectroscopy}

The FTIR spectra over the range of $400-4000 \mathrm{~cm}^{-1}$ for DOX, Compritol 888 ATO, and physical mixture of DOX and Compritol 888 ATO are shown in fig. 5.

Important peaks at $3453.59 \mathrm{~cm}^{-1}$ and $3286.90 \mathrm{~cm}^{-1}$ appeared the characteristic peak of $\mathrm{NH} 3+$ Stretching, $\mathrm{O}-\mathrm{H}$ stretching, H-Bonding, at $2883.64 \mathrm{~cm}^{-1}$ alkane $\mathrm{CH}$ group stretch, at $1736.32 \mathrm{~cm}^{-1}$ and 1728.94 $\mathrm{cm}^{-1}$ carboxylic acid $\mathrm{C}=0$ stretch, at $1173.46 \mathrm{~cm}^{-1}$ and $1104.88 \mathrm{~cm}^{-1}$ C-O stretches. In the FTIR spectrum of physical mixtures of DOX and Compritol 888 ATO, these characteristic peaks of DOX were also observed without any distinct changes. This reality confirmed that no chemical reaction had taken place between the drug and the polymer. Compritol 888 ATO was selected as the lipid for preparing solid lipid nanosuspension based on a drug lipid solubility and drug lipid compatibility analysis.

\section{Differential scanning calorimetry (DSC)}

Compatibility studies of drug polymers are very essential before formulation design DSC helps to provide useful information about crystallite and amorphism of the prepared sample. Perkin-Elmer Diamond DSC was used to investigate the melting and recrystallization behavior of the SLNs. DSC thermograms of doxorubicin, doxorubicinloaded SLNs, Blank (Placebo) SLNs, physical mixture (DOX and lipid), Compritol 888 ATO, are presented in fig. 6.

Doxorubicin exhibited an endotherm corresponding to its MP at $\sim 235^{\circ} \mathrm{C}$. The thermal curve of the Compritol 888 ATO exhibited an endothermic peak at $\sim 69{ }^{\circ} \mathrm{C}$. Whereas, physical mixture (DOX and lipid) exhibited a sharp endothermic peak at $\sim 68^{\circ} \mathrm{C}$ and a very small peak was found at about $234{ }^{\circ} \mathrm{C}$, which was present in DOX. The melting endotherm of Compritol 888 ATO in blank SLNs and DOX SLN formulation was observed at $68.03{ }^{\circ} \mathrm{C}$ and $66.06{ }^{\circ} \mathrm{C}$, respectively. DSC thermograms of a physical mixture of DOX+Lipid and bulk lipid exhibited a melting endotherm corresponding to the melting of DOX at $\sim 65^{\circ} \mathrm{C}$, which was present in DOX-SLNs. However, the DOX peak was lost in DOX SLNs [fig. 6. (a) and i(e)]. It could be deduced that DOX in SLNs was in an amorphous state [28].

\section{$\mathrm{X}$-ray diffraction (XRD)}

XRD patterns of DOX, Compritol 888 ATO, the physical mixture (drug+lipid), blank SLNs, and DOX-SLNs are shown in fig. 7. 
Shah et al.

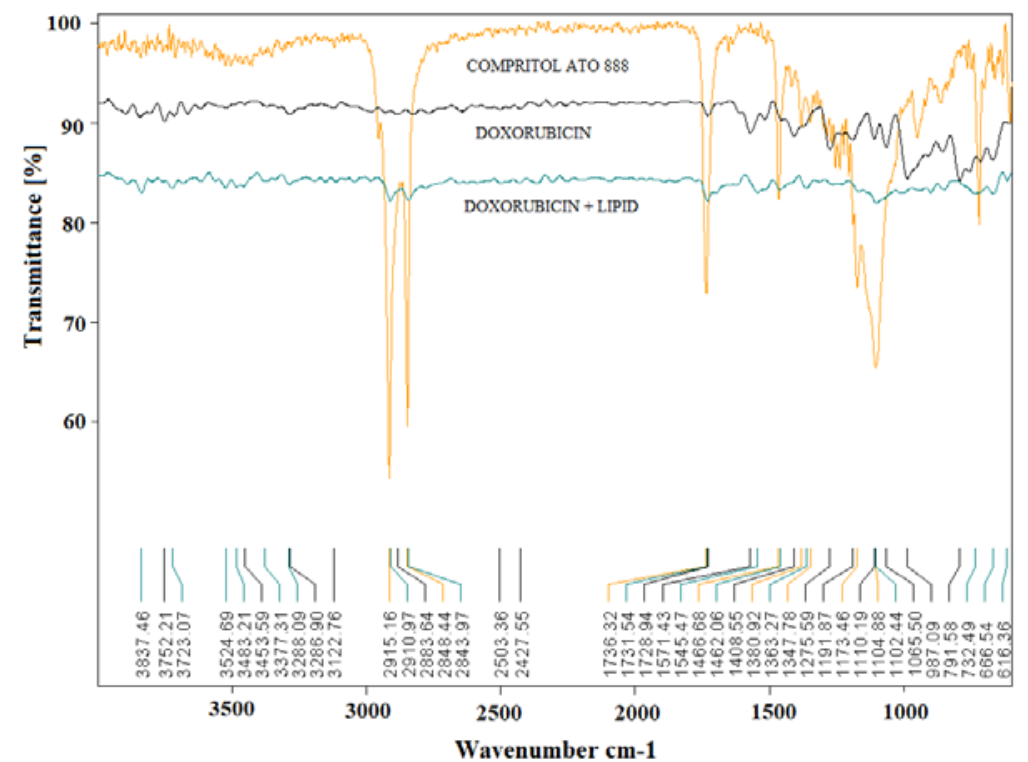

Fig. 5: IR spectra of DOX, compritol 888 ATO 88, and physical mixtures of DOX and compritol 888 ATO

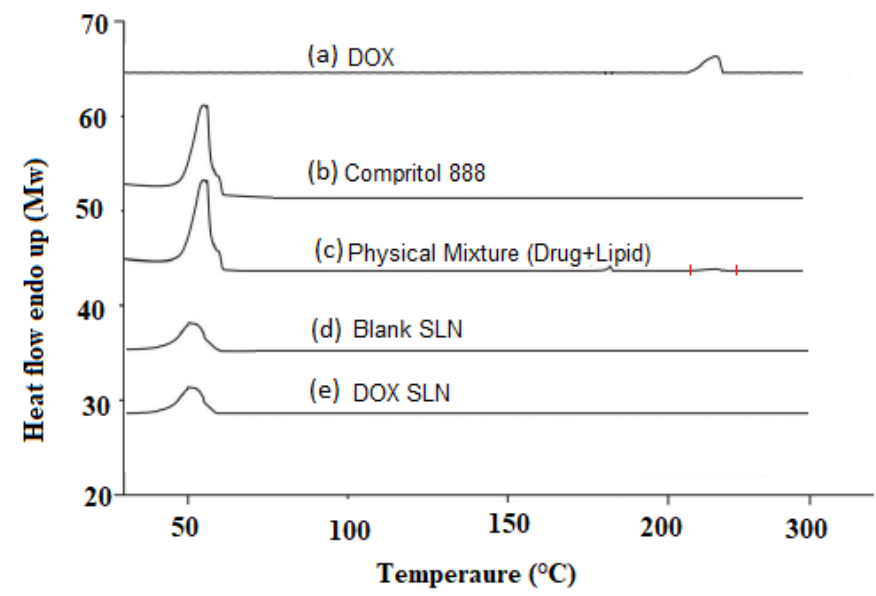

Fig. 6: DSC Thermogram (a) doxorubicin (DOX) (b) Compritol 888 (c) Physical mixture (DOX+Lipid) (d) Blank SLNs (e) DOX SLNs

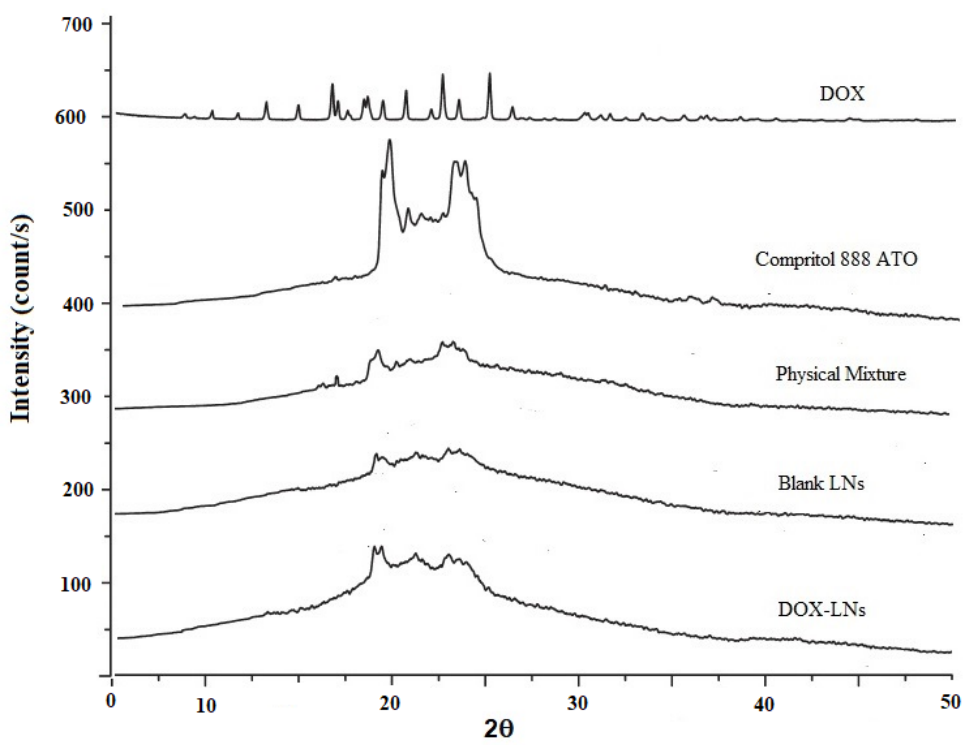

Fig. 7: X-ray diffraction patterns DOX, Compritol 888 ATO, the physical mixture (drug+lipid), blank SLNs, and DOX-SLNs 
At scattered angles, DOX's XRD patterns displayed sharp peaks, ranging from $15^{\circ}$ to $25^{\circ}$, suggesting their crystalline existence. Light diffraction peaks of DOX were observed in the physical mixture at around $16^{\circ}$, showing incomplete dissolution and the presence of a crystalline state of both drugs in bulk lipid. The blank SLN and DOX-SLNs XRD patterns were wider and much weaker than the bulk lipid. There were no characteristic peaks in
DOX-SLNs for DOX, however, indicating the amorphous state of DOX. The XRD result of these DOX-SLNs was following that of the DSC.

\section{Particle size, PDI, and zeta potential}

Particle size distribution and zeta potential curve of optimized formulation (V-0) were shown in fig. $8(a, b)$, respectively

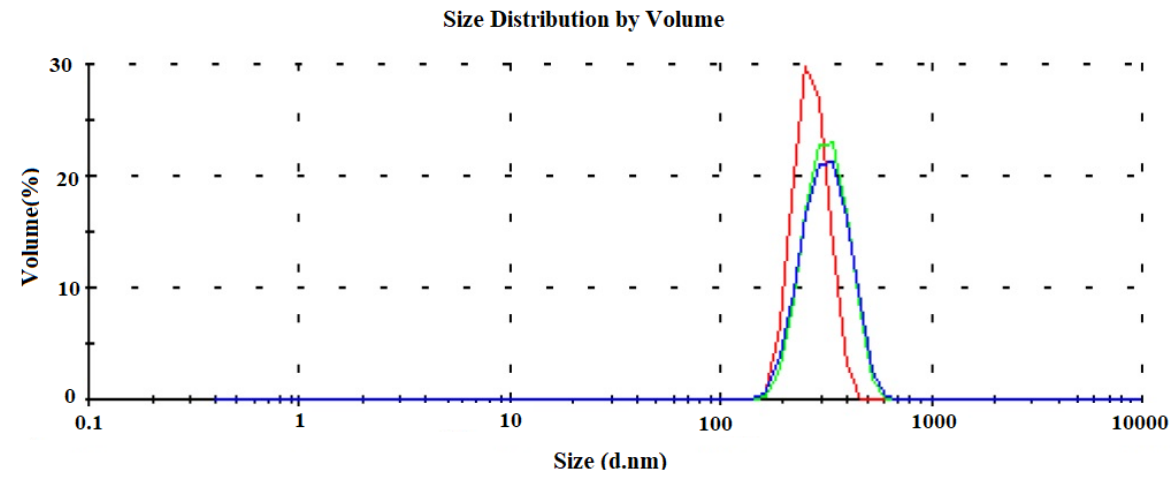

\begin{tabular}{|lll|}
\hline Record 1: $\mathrm{V}-\mathrm{O} a$ & Record 2: $\mathrm{V}-\mathrm{Ob} \quad$ Record 3: $\mathrm{V}-\mathrm{O} \mathrm{c}$ \\
\hline
\end{tabular}

(a) Plot for the size distribution vs number for batch $\mathrm{V}-\mathrm{O}(\mathrm{n}=3)$

\section{Zeta Potential Distribution}

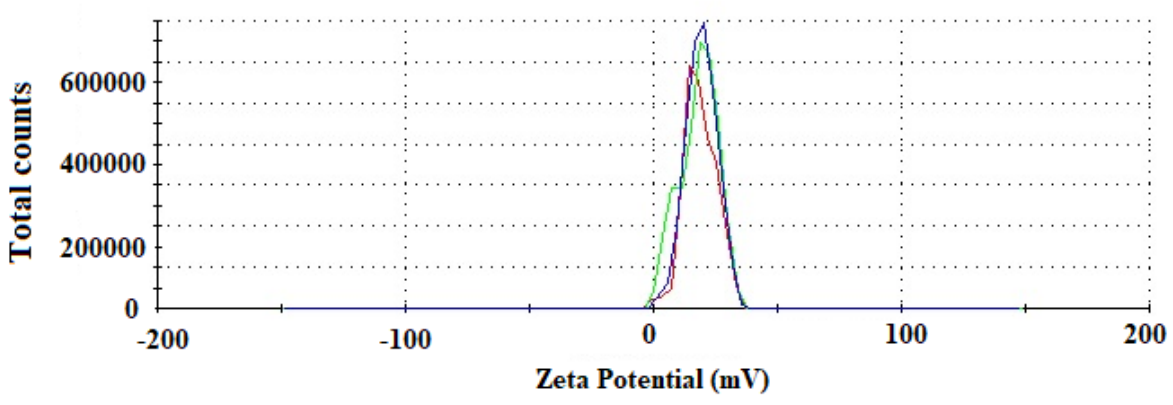

Plot of zeta potential di

Fig. 8: (a) Particle size distribution curve, (b) zeta potential curve of optimized formulation

The average particle size of the optimized formulation was 167.47 $\pm 6.09 \mathrm{~nm}$, which was found to be sufficient for nasal pathway brain targeting. In essence, PDI is the ratio of the standard deviation to the mean size of particles. A PDI value of 0.3 or less suggests particle size uniformity. In this case, the PDI value of the formed SLNs was found to be $0.23 \pm 0.02$, suggesting uniformity in particle size. In the dispersion of SLNs, the zeta potential shows the degree of charge present on suspended particles. A suitably high zeta potential value $(30 \mathrm{mV}$ to- $30 \mathrm{mV})$ confers stability since aggregation is resisted by particles. The zeta potential $(24.1 \mathrm{mV})$ value of the optimized formulation of SLNs showed good stability [29].

\section{Morphological study}

Morphological inspection of DOX SLNs using TEM analysis showed that SLNs were spherical (fig. 9) and were in the size range of 150-200 $\mathrm{nm}$, which was based on the dynamic light scattering theory, in further agreement with the size distribution performed using Zetasizer. From fig. 9 It was noted that the particles of the SLNs had a uniform distribution of size, confirmed by Zetasizer, showing a PDI value below 0.25 , suggesting no aggregation.

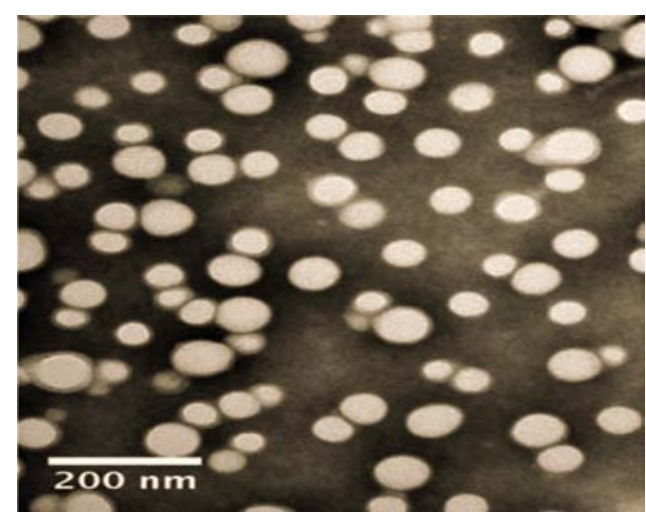

Fig. 9: TEM image of optimized DOX-SLNs formulation

\section{Entrapment efficiency (\%)}

The entrapment efficiency of optimized DOX SLNs formulation was found to be $75.3 \pm 2.79$. 


\section{$p H$}

The optimized formulation of DOX SLNs showed a $\mathrm{pH}$ value of $5.86 \pm 0.35$, which was within the standard $\mathrm{pH}$ range of 5-6.5 for human nasal mucosa and thus does not cause nasal inflammation when administered intranasally [30].

\section{Viscosity}

The viscosity values for the optimized formulation of DOX-loaded SLNs were $39.5 \pm 0.93 \mathrm{cP}$, which was lower than $50 \mathrm{cP}$, thus ensuring strong intranasal administration tolerance. Formulation with a higher viscosity value often results in increased residence time but faces administration difficulties and may display decreased absorption due to reduced drug diffusion from the formulation, whereas systems with lower viscosity can be easily administered but have to face faster MCC, thus reducing the contact time of the mucosa formulation applied [31].

\section{In vitro drug release study}

A comparative in vitro release analysis was conducted between the commercialized liposomal preparation of DOX and the optimized formulation of DOX-SLNs (V-0). In figure.10. the comparative dissolution profile was shown. The DOX-SLNs dissolution profile showed an initial burst release, followed by a gradual release. The initial burst release may be attributed to the presence of free drugs on the surface of particles in the external phase and adsorbed drugs, whereas the slow release may be due to the encapsulated drug inside the lipid matrix [32].

The optimized DOX SLNs showed an initial release of $8.43 \pm 1.49$ percent, compared to $8.13 \pm 1.52$ percent after $1 \mathrm{~h}$ for commercially available DOX liposomal preparation. Optimized DOX SLNs subsequently showed sustained drug release with a cumulative drug release of $89.35 \pm 3.27$ percent over $24 \mathrm{~h}$, while Marketed DOX Liposomal preparation showed $94.9 \pm 4.64$ percent over $24 \mathrm{~h}$. An f2 parameter is widely used to determine the similarity of two dissolution profiles. To show the similarity between two dissolution profiles, a public norm of $\mathrm{f} 2$ value between $50-100$ is used. In our case, the similarity factor $\mathrm{f} 2=98.33$ indicates that the similarity between the optimized DOX-SLNs formulation dissolution profile and the marked Dox liposomal preparation is identical.
Analysis of kinetics and mechanism of drug release for optimized DOX SLNs

Optimized doxorubicin-loaded lipid nanosuspension (DOX SLNS) formulations were fitted into various kinetic models. The correlation coefficient $\left(\mathrm{R}^{2}\right)$ and exponent values of various kinetic models are shown in table 8.

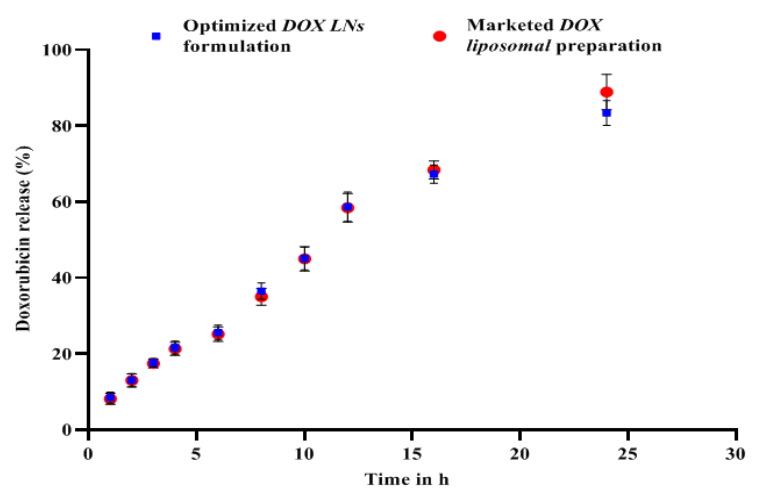

Fig. 10: Comparison of \% in vitro drug release profile of optimized DOX-LNs with marketed DOX liposomal preparation

The results showed that the kinetic models that could be used to characterize the release characteristics of doxorubicin-loaded lipid nanosuspension (DOX-SLNs) formulations were better suited to the zero-order model. The zero-order kinetics model had the highest correlation coefficients $\left(R^{2}=0.982\right)$ compared to other kinetic models. Diffusion of the drug from the lipid matrix has therefore been proposed to be the possible mechanism of action. The value of release exponent "n" was found to be 0.4597 in the KorsmeyerPeppas model, which seems to suggest the process of release regulated by diffusion, so-called Fickian diffusion. Similar results have been observed in Shazly [33], who prepared ciprofloxacinloaded SLNs.

Table 8: Release kinetic models for the optimized DOX-SLNs formulation

\begin{tabular}{|c|c|c|c|c|c|c|c|c|c|c|}
\hline \multirow{2}{*}{$\begin{array}{l}\text { Optimized } \\
\text { DOX-SLNS }\end{array}$} & \multicolumn{2}{|c|}{ Zero-order } & \multicolumn{2}{|c|}{ First-order } & \multicolumn{2}{|c|}{ Higuchi model } & \multicolumn{2}{|c|}{ Hixon Crowell model } & \multicolumn{2}{|c|}{ Korsmeyer-peppas } \\
\hline & $\mathbf{R}^{2}$ & $\mathrm{~K}_{0}\left(\mathrm{~h}^{-1}\right)$ & $\mathbf{R}^{2}$ & $K_{0}\left(h^{-1}\right)$ & $\mathbf{R}^{2}$ & $K_{0}\left(h^{-1}\right)$ & $\mathbf{R}^{2}$ & $\mathrm{~K}_{0}\left(\mathrm{~h}^{-1}\right)$ & $\mathbf{R}^{2}$ & n value \\
\hline $\mathrm{V}-\mathrm{O}$ & 0.982 & 3.64 & 0.956 & 0.03 & 0.973 & 21.43 & 0.98 & 0.09 & 0.8561 & 0.4597 \\
\hline
\end{tabular}

\section{Stability studies}

Table 9: Characteristics of DOX-SLNs after 3-month stability studies at different conditions

\begin{tabular}{|c|c|c|c|c|c|}
\hline \multirow{2}{*}{$\begin{array}{l}\operatorname{Temp}\left({ }^{\circ} \mathrm{C}\right) / \\
\operatorname{RH}(\%)\end{array}$} & \multirow[t]{2}{*}{ Time (month) } & \multicolumn{4}{|c|}{ Characteristics parameter } \\
\hline & & Particle size (nm) ${ }^{a}$ & PDI & Zeta potential $(\mathrm{mV})^{\mathrm{a}}$ & Entrapment efficiency (\%) \\
\hline \multirow[t]{3}{*}{$4 \pm 2$} & 0 & $167.47 \pm 6.09$ & $0.23 \pm 0.02$ & $24.1 \pm 1.7$ & $75.3 \pm 2.79$ \\
\hline & 1 & $169.94 \pm 5.82$ & $0.272 \pm 0.03$ & $23.3 \pm 1.2$ & $75.54 \pm 1.20$ \\
\hline & 3 & $171.65 \pm 2.54$ & $0.284 \pm 0.05$ & $22.6 \pm 1.5$ & $75.23 \pm 1.94$ \\
\hline \multirow[t]{3}{*}{$25 \pm 2 / 60 \pm 5$} & 0 & $167.47 \pm 6.09$ & $0.23 \pm 0.02$ & $24.1 \pm 1.7$ & $75.3 \pm 2.79$ \\
\hline & 1 & $167.21 \pm 3.71$ & $0.226 \pm 0.02$ & $23.2 \pm 1.2$ & $75.65 \pm 1.24$ \\
\hline & 3 & $174.76 \pm 5.57$ & $0.287 \pm 0.03$ & $22.5 \pm 1.3$ & $74.78 \pm 1.98$ \\
\hline \multirow[t]{3}{*}{$40 \pm 2 / 75 \pm 5$} & 0 & $167.47 \pm 6.09$ & $0.23 \pm 0.02$ & $24.1 \pm 1.7$ & $75.3 \pm 2.79$ \\
\hline & 1 & $174.45 \pm 2.81$ & $0.285 \pm 0.04$ & $20.2 \pm 1.9$ & $75.39 \pm 2.85$ \\
\hline & 3 & $355.35 \pm 3.98$ & $0.422 \pm 0.03$ & $16.6 \pm 2.3$ & $74.53 \pm 1.27$ \\
\hline
\end{tabular}

aData was expressed as mean $\pm \mathrm{SD}, \mathrm{n}=3$; $\mathrm{P}<0.05$ (measurements obtained at each point in time $(1 \mathrm{and} 3 \mathrm{mo}$ ) at the same storage condition were compared with measurements obtained at that storage condition at zero months. There has also been a comparison of various parameters between different temperatures), No substantial difference in particle size was observed when stored at $4 \pm 2{ }^{\circ} \mathrm{C}$ (refrigerator) and $25 \pm 2{ }^{\circ} \mathrm{C} / 60 \pm 5$ percent $\mathrm{RH}$, but when stored at $40 \pm 21 \mathrm{C} / 75 \pm 5$ percent RH due to aggregation, the particle size increased $(\mathrm{P}<0.05$, table 9$)$. There was a substantial shift between $4 \pm 2{ }^{\circ} \mathrm{C}$ (refrigerator) and $25 \pm 2{ }^{\circ} \mathrm{C} / 60 \pm 5$ percent $\mathrm{RH}$, but the zeta potential fell to $40 \pm 21 \mathrm{C} / 75 \pm 5$ percent $\mathrm{RH}(\mathrm{P}<0.05)$. Zeta potential plays an important role in physical stability [35]. This may be due to lipid coating dissolution that contributes to particle aggregation.

\section{CONCLUSION}

A lipid nanoparticulate drug delivery system (SLNs) of doxorubicin was proposed for brain targeting through intranasal delivery in the present research work. SLNs were prepared by the homogenization and ultra-sonication process and evaluated for particle size, particle size distribution (PDI), zeta potential, entrapment efficiency, in vitro release, and stability studies. It was noticed that all measurements 
were in an acceptable range. To prepare solid lipid nanoparticles of reproducible sizes in the range of 137.3 to $306.8 \mathrm{~nm}$ by addressing the effects of processing parameters, high-speed homogenization followed by ultrasonication technique was used. The $3^{3}$ factorial design application proved to be a useful method for optimizing DOXloaded SLNs. Using a factorial design, the required formulation composition can be selected to obtain DOX-loaded SLNs smaller than $200 \mathrm{~nm}$, depending on the application of the brain targeting system through intranasal delivery. The use of $3^{3}$ full-factor designs allowed the production of an appropriate formulation using the minimum amount of raw materials and a minimum of time. In vitro drug release over $24 \mathrm{~h}$ was found to be $89.35 \pm 3.27$ percent, suggesting a controlled and sustained DOX-SLN release profile. No major changes in particle size, zeta potential, and entrapping efficiency were observed in the stability studies at $4 \pm 2{ }^{\circ} \mathrm{C}$ (refrigerator) and $25 \pm 2$ ${ }^{\circ} \mathrm{C} / 60 \pm 5$ percent $\mathrm{RH}$ for up to $3 \mathrm{mo}$. This study, therefore, demonstrated the usefulness of SLNs for the delivery of doxorubicin via the intranasal (i. $n$ ) route to the brain.

\section{ACKNOWLEDGMENT}

The Valuable assistance from Amneal Pharmaceuticals Ltd. (India, Ahmedabad) and Gattefosse Pvt. Ltd. (Mumbai, India) is gratefully remembered for supplying the drug and lipid-based excipients, respectively. Dr. Girish K. Jani, former principal of the SSR College of Pharmacy, Silvassa, DNH, India, is very grateful to the authors for their assistance and helpful suggestions.

\section{FUNDING}

Nil

\section{AUTHORS CONTRIBUTIONS}

Virag A. Shah has performed experiments and prepared manuscript draft, Dr. Jayvadan K. Patel guided and supervised the research work.

\section{CONFLICT OF INTERESTS}

The author declared that there is no conflict of interest regarding the publication of the paper

\section{REFERENCES}

1. Zubareva A, Shcherbinina T, Varlamov VP, Svirshchevskaya E. Biodistribution of doxorubicin-loaded succinyl chitosan nanoparticles in mice injected via intravenous or intranasal routes. PCACD 2014;19:145-54.

2. Mussi V, Silva C. New approach to improve encapsulation and antitumor activity of doxorubicin-loaded in solid lipid nanoparticles. Eur J Pharm Sci 2013;48:282-90.

3. Oliveira S, Mussi V. $\alpha$-Tocopherol succinate improves encapsulation and anticancer activity of doxorubicin-loaded in solid lipid nanoparticles. Colloids Surf B 2016;140:246-53.

4. Lala RR, Shinde AS, Nandvikar NY. Solid lipid nanoparticles: a promising approach for combinational drug therapy in cancer. Int J Appl Pharm 2018;10:17-22.

5. Xiong P, Ding A, Su Z, Shen G, Chen Y, Zhang S. The efficacy and hyperthermic release of doxorubicin from liposomal doxorubicin hydrochloride in rabbits VX2 tumors. Int J Hyperthermia 2015;31:900-8.

6. Khatak S, Dureja H. Structural composition of solid lipid nanoparticles for invasive and non-invasive drug delivery. Curr Nanomaterials 2017;2:129-53.

7. Patel S, Chavhan S. Brain targeting of risperidone-loaded solid lipid nanoparticles by the intranasal route.J Drug Target 2011;19:468-74.

8. Shah M, Pathak K. Development and statistical optimization of solid lipid nanoparticles of simvastatin by using $2^{3}$ full-factorial design. AAPS PharmSciTech 2010;11:489-96.

9. Gambhire S, Bhalekar R, Gambhire M. Statistical optimization of dithranol-loaded solid lipid nanoparticles using factorial design. Braz J Pharm Sci 2011;47:503-11.

10. Yasir M, Sara S. Solid lipid nanoparticles for a nose to brain delivery of haloperidol: in vitro drug release and pharmacokinetics evaluation. Acta Pharm Sin B 2014;4:454-63.
11. Bilati U, Allemann E, Doelker E. Development of a nanoprecipitation method intended for the entrapment of hydrophilic drugs into nanoparticles. Eur J Pharm Sci 2005;25:67-75.

12. Dong $\mathrm{Y}, \mathrm{Ng} \mathrm{W}$, Shen $\mathrm{S}$, Kim S, Tan K. Solid lipid nanoparticles: continuous and potential large-scale nanoprecipitation production in static mixers. Colloids Surf B 2012;94:68-72.

13. Costa P, Lobo S. Modeling and comparison of dissolution profiles. Eur J Pharm Sci 2001;13:123-33.

14. Gan L, Gao YP, Zhu CL. Novel pH-sensitive lipid-polymer composite microspheres of 10-hydroxycamptothecin exhibiting colon-specific biodistribution and reduced systemic absorption. J Pharm Sci 2013;102:1752-9.

15. Blasi P, Giovagnoli S, Schoubben A. Lipid nanoparticles for brain targeting formulation optimization. Int J Pharm 2011;419:287-95.

16. Bose S, Du Y, Takhistov P, Michniak Kohn B. Formulation optimization and topical delivery of quercetin from solid lipidbased nanosystems. Int J Pharm 2013;441:56-66.

17. Rajkumar M, Bhise B. Carbamazepine-loaded porous microspheres for short-term sustained drug delivery. J Young Pharm 2010;2:7-14.

18. Rahman Z, Zidan S, Khan A. Non-destructive methods of characterization of risperidone solid lipid nanoparticles. Eur J Pharm Biopharm 2010;76:127-37.

19. Borgia L, Regehly M, Sivaramakrishnan R. Lipid nanoparticles for skin penetration enhancement-correlation to drug localization within the particle-matrix as determined by fluorescence and parelectric spectroscopy. J Controlled Release 2005;110:151-63.

20. Cai S, Yang Q, Bagby R, Forrest L. Lymphatic drug delivery using engineered liposomes and solid lipid nanoparticles. Adv Drug Delivery Rev 2011;10:901-8.

21. Sarkar T, Ahmed B. Development and in vitro characterization of chitosan loaded paclitaxel nanoparticle. Asian J Pharm Clin Res 2016;9:145-8.

22. Hooda A, Nanda M, Jain V, Kumar P, Rathee. Optimization and evaluation of gastroretentive ranitidine $\mathrm{HCl}$ microspheres by using design expert software. Int J Biol Macromol 2012;51:691-700.

23. Mahajan A, Kaur S. Design, formulation, and characterization of stearic acid-based solid lipid nanoparticles of candesartan cilexetil to augment its oral bioavailability. Asian J Pharm Clin Res 2018;11:344-50.

24. Malakar J, Nayak K, Pal D. Development of cloxacillin loaded multiple-unit alginate-based floating system by an emulsiongelation method. Int J Biol Macromol 2012;50:138-47.

25. Reddy H, Murthy R. Etoposide-loaded nanoparticles made from glyceride lipids: formulation, characterization, in vitro drug release, and stability evaluation. AAPS PharmSciTech 2005;6:158-66.

26. Thakkar P, Desai L, Parmar P. Application of Box-Behnken design for optimization of formulation parameters for nanostructured lipid carriers of candesartan cilexetil. Asian J Pharm 2014;8:81-9.

27. Shah M, Pathak K. Development and statistical optimization of solid lipid nanoparticles of simvastatin by using $2^{3}$ full-factorial design. AAPS PharmSciTech 2010;11:489-96.

28. Subedi K, Kang W, Choi K. Preparation and characterization of solid lipid nanoparticles loaded with doxorubicin. Eur J Pharm Sci 2009;37:508-13.

29. Zhuang Y, Li N, Wang M. Preparation and characterization of vinpocetine loaded nanostructured lipid carriers (NLC) for improved oral bioavailability. Int J Pharm 2010;394:179-85.

30. Abdelbary G, Fahmy H. Diazepam-loaded solid lipid nanoparticles: design and characterization. AAPS PharmSciTech 2009;10:211-9.

31. Arora P, Sharma S, Garg S. Permeability issues in nasal drug delivery. Drug Discovery Today 2002;7:967-75.

32. Furubayashi T, Inoue D, Kamaguchi A, Higashi Y, Sakane T. Influence of formulation viscosity on drug absorption following nasal application in rats. Drug Metab Pharmacokinet 2007;22:206-11.

33. Joshi S, Patel S, Belgamwar S. Solid lipid nanoparticles of ondansetron $\mathrm{HCl}$ for intranasal delivery: development, optimization, and evaluation. J Mater Sci Mater Med 2012;23:2163-75. 
34. Shazly A. Ciprofloxacin controlled-solid lipid nanoparticles: characterization, in vitro release, and antibacterial activity assessment. BioMed Res Int 2017;2017:1-9.
35. Aher S, Malsane T, Saudagar R. Nanosuspension: an overview. Int J Curr Pharm Res 2017;9:19-23. 